\title{
Monte-Carlo calculation of fission process for neutron-induced typical ac- tinide nuclei fission
}

\author{
Zheng Wei ${ }^{1,2, *}$, Changqi $\mathrm{Liu}^{1}$, Chao $\mathrm{Han}^{1}$, Zeen $\mathrm{Yao}^{1,2}$, Yu Zhang ${ }^{1,2}$, Junrun Wang ${ }^{1,2}$, Xiaolong Lu $^{1,2}$, Xiaodong Su ${ }^{1,2}$, \\ and Dapeng $\mathrm{Xu}^{1,2}$ \\ ${ }^{1}$ School of Nuclear Science and Technology, Lanzhou University, 730000, Lanzhou, China \\ ${ }^{2}$ Engineering Research Center for Neutron Application, Ministry of Education, 730000, Lanzhou, China
}

\begin{abstract}
A global potential-driving model with well-determined parameters is proposed by uniting the empirical asymmetric fission potential and the empirical symmetric fission potential, which can precisely calculate the pre-neutron-emission mass distributions for neutron-induced actinide nuclei fission. Based on the developed potential-driving model, Monte-Carlo code calculates the characteristics of fission reaction process for neutron-induced ${ }^{241} \mathrm{Am}$ fission. Typical calculated results, including yields, kinetic energy distributions, fission neutron spectrum and decay $\gamma$-ray spectrum, are compared with experimental data and evaluated data. It shows that the Monte-Carlo calculated results agree quite well with the experiment data, which indicate that MonteCarlo code with the developed potential-driving model can reproduce and predict the characteristics of fission reaction process at reasonable energy ranges. Given the well predictions on the characteristics of fission reaction process, Monte-Carlo code with the developed potential-driving model can guide for the physical design of nuclear fission engineering.
\end{abstract}

\section{Introduction}

Fission characteristics of neutron-induced actinides fission are important for reactor applications. The neutroninduced fission data of ${ }^{232} \mathrm{Th},{ }^{233} \mathrm{U},{ }^{235} \mathrm{U},{ }^{238} \mathrm{U}$ and ${ }^{239} \mathrm{Pu}$ has a significant application in light-water reactors [1, 2], molten salt reactor [3], hybrid subcritical systems [4], accelerator driven systems (ADS) [5]. With the accumulation of long lived Minor Actinides (MAs), such as ${ }^{237} \mathrm{~Np}$, ${ }^{241} \mathrm{Am}$ and ${ }^{244} \mathrm{Cm}$, post-processing of spent nuclear fuel has aroused wide attention around the world. Their long half-life and high-level of radioactivity have a strong influence on sustainable development of nuclear power. ${ }^{241} \mathrm{Am}$ is in particular considered as one of the most important actinides in reprocessing of spent nuclear fuel [6].

We have developed a global potential-driving model by uniting the empirical asymmetric fission potential and the empirical symmetric fission potential to precisely calculate the pre-neutron-emission mass distributions for neutron-induced actinide nuclei fission [7]. In this work, based on the developed potential-driving model, MonteCarlo code calculates the characteristics of fission reaction process for neutron-induced typical actinide nuclei ${ }^{241} \mathrm{Am}$ fission. Important and detailed information, such as the fission yields, kinetic energy distributions, fission neutron spectrum and decay $\gamma$-ray spectrum, are compared with the nuclear data tables and experimental data, and the applicability of the potential-driving model is evaluated.

\section{Potential-driving model}

In the processes of neutron-induced actinide nuclei fission, the specific compound nucleus takes place deformation, when the deformation is large enough, the specific compound nucleus breaks up into two fragments at the scission point. In this work, we attempt to propose empirical fission potentials to drive to calculate the preneutron-emission mass distribution [7]. The potentialdriving model can describe the driving potential $D$ of a fissile system around the scission point by sum of a symmetric fission potential and asymmetric fission potentials. The driving potential $D$ can be expressed as [7]

$$
\begin{aligned}
D= & U_{\text {sym }}\left(A_{f}\right)+U_{\text {asym }}\left(A_{f}\right) \\
= & D\left(A_{\text {sym }}\right) \cdot \exp \left[-\frac{\left(A_{f}-A_{\text {sym }}\right)^{2}}{2 \delta_{\text {sym }}^{2}}\right]+\sum_{i} \eta_{i} \cdot D\left(A_{\text {asym }, i}\right) \\
& \cdot \exp \left[-\frac{\left(A_{f}-A_{\text {asym }, i}\right)^{2}}{2 \delta_{\text {asym }, i}^{2}}\right] .
\end{aligned}
$$

where $U_{\text {sym }}\left(A_{f}\right)$ and $U_{\text {asym }}\left(A_{f}\right)$ are the symmetric and asymmetric fission potentials. The variable $A_{f}$ denotes the mass number of the fission fragment. $D\left(A_{\text {sym }}\right)$ and $D\left(A_{\text {asym }, i}\right)$ are the strength of the symmetric and asymmetric fission potentials. $A_{s y m}$ and $A_{a s y m, i}$ are the peak positions for the symmetric and asymmetric fission potentials, respectively.

\footnotetext{
*e-mail: weizheng@1zu.edu.cn
} 
Generally, the pre-neutron-emission mass distribution of fission fragments can be described as [7]

$$
F\left(A_{f}\right)=C \times\left[\frac{U_{\text {asym }}\left(A_{f}\right)}{T\left(A_{C N}\right)}+\omega \cdot \frac{U_{\text {sym }}\left(A_{f}\right)}{T\left(A_{C N}\right)}\right] .
$$

where $C$ is a normalization constant. $T\left(A_{C N}\right)$ is the temperature of the specific compound nucleus. The weight $\omega\left(E_{n}, Z, A\right)$ defines the relative contribution of each driving potential and it is strongly dependent on peak-to-valley ratio.

In order to verify the reliability of the potential-driving model, we have calculated pre-neutron emission mass distributions for neutrons induced fission of ${ }^{232} \mathrm{Th},,{ }^{238} \mathrm{U}$, ${ }^{237} \mathrm{~Np}$ and ${ }^{239} \mathrm{Pu}$, as shown in Fig. 1. The calculated results agree well with the experimental data [8-12] for neutron-induced actinide nuclei fission, which indicates that the potential-driving model can reproduce the measured results at different incident energies. In particular, taking into account the energy dependence of evaporation neutrons from the reactions [13, 14], the potential-driving model can calculate pre-neutron emission mass distributions for the reaction with incident neutron energies up to $160 \mathrm{MeV}$. Given the good agreement with the experimental data, the potential-driving model should predict well the mass distributions for the reaction at unmeasured energies.
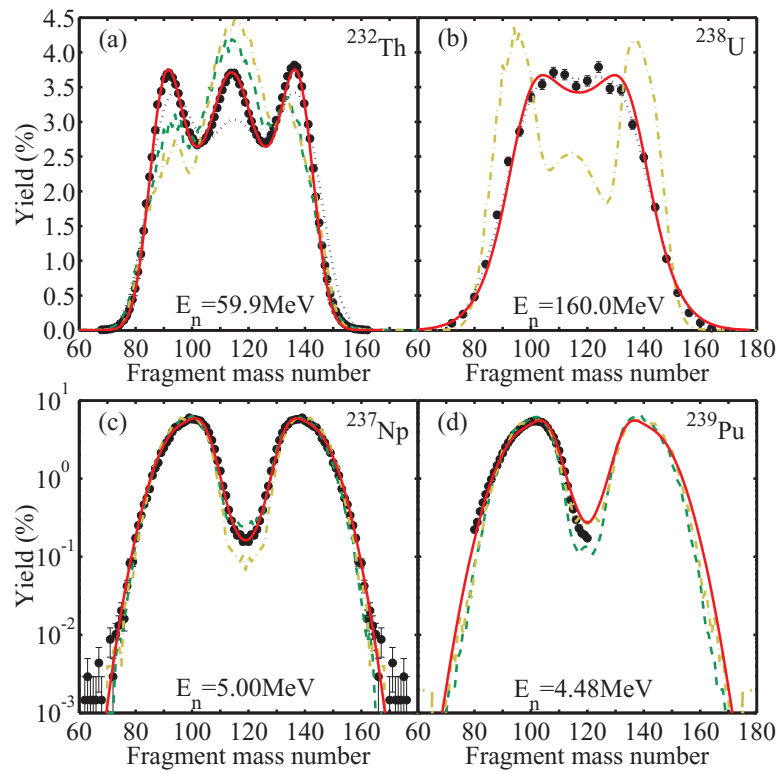

Figure 1. (color online). Pre-neutron emission fission-fragment mass distribution for neutron-induced typical actinide nuclei fission at different incident energies. The solid circles denote the experimental data, the red curves denote the calculated data from the potential-driving model, the green dashed curves denote the calculated results of the GEF-2014-V2-1 code [15], the yellow dot-dash curves denote the calculated results of the TALYS-1.8 code [16], and the blue dotted curves denote the calculated results of the PYF-2.2 code [17], respectively.

\section{Monte Carlo simulation of ${ }^{241} \mathrm{Am}(\mathbf{n}, \mathbf{f})$ reaction}

In the processes of neutron-induced actinide nuclei fission, the highly excited primary fission fragments are deexcited by the emission of prompt neutrons, and followed by prompt $\gamma$-rays to form the primary fission products. The primary fission products are usually highly neutron rich and unstable, and gradually evolve to the secondary fission products through the emission of delayed neutrons and the radioactive $\gamma$ decay.

Based on the potential-driving model, Monte Carlo code, Geant4, simulates the $14 \mathrm{MeV}$ fast neutron-induced actinide nuclei fission $[7,18]$. The fission process involves two continuous processes (a) the creation of fission reaction and (b) the unstable fission fragments refund excitation process, which are taken in account in the Geant 4 physical model [19].

The calculated mass distributions of independent yields and cumulative yields from ${ }^{241} \mathrm{Am}(\mathrm{n}, \mathrm{f})$ reaction are shown in Fig. 2, which are compared with experimental data $[20,21]$ as well as the evaluated nuclear data from ENDF/B-VII.1. Compared with the G4ParaFissionModel [19], the calculated mass distributions results from the potential-driving model are better agreement with the experiment data and ENDF/B-VII.1 data. From the Fig. 2, one can see that Geant 4 simulates the processes of neutron-induced actinide nuclei fission based on the potential-driving model, and the calculated yields distributions are agreed quite well with experimental data and evaluated data. The potential-driving model shows a significant advance with regard to accuracy.
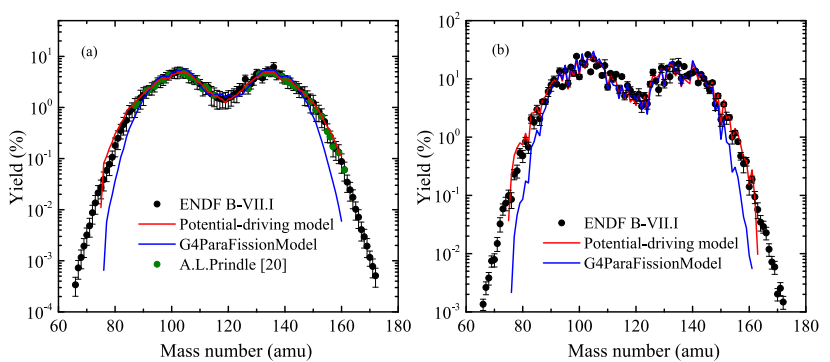

Figure 2. (color online). The comparison between the simulated data and the recommended data. The mass chain yield versus mass number for (a) independent yield and (b) cumulative yield of ${ }^{241} \mathrm{Am}(\mathrm{n}, \mathrm{f})$ reaction. The red lines denote calculated results from the potential-driving model, the blue lines denote calculated results from G4ParaFissionModel [19], and the color dots and black dots denote experimental and evaluated data, respectively.

In the processes of neutron-induced ${ }^{241} \mathrm{Am}$ nuclei fission, the fissioning nucleus splits into different light fission-fragments. The fission of a heavy nucleus releases about $200 \mathrm{MeV}$, and the large part of energy is spent as fission fragment kinetic energy. As one of the most significant physical quantity, it is closely related to deformation of fission nucleus and potential energy at the scission point. 
Fig. 3 shows the relationship between the fissionfragment average kinetic energy $(\overline{T K E})$ and the fissionfragment mass number for ${ }^{241} \mathrm{Am}(\mathrm{n}, \mathrm{f})$ reaction. According to the fission-fragment average kinetic energy distribution, light fission-fragment products have higher kinetic energy, and heavy fission-fragment products with lower kinetic energy, owing to momentum conservation law. In reactor engineering design, the stopping process transforms the fission-fragment kinetic energy to thermal energy, which is very important for reactor engineering design and selection of reactor wall material.

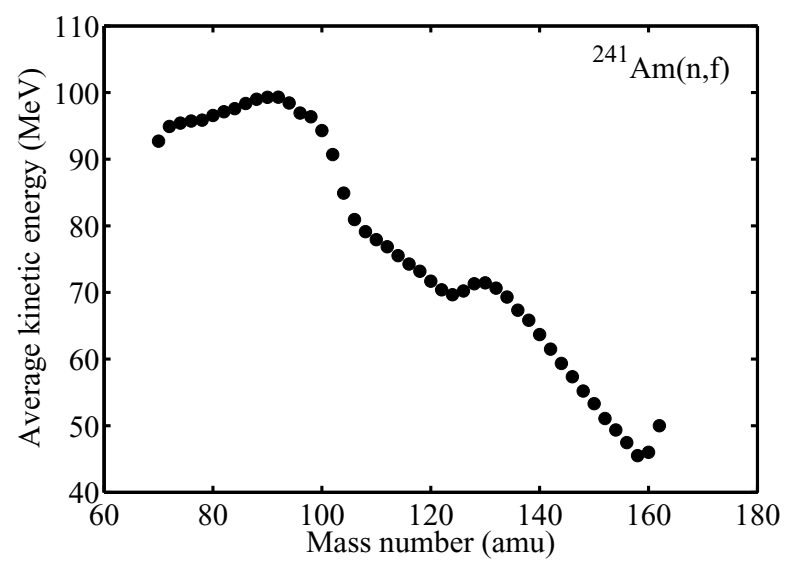

Figure 3. The relationship between the fission-fragment average kinetic energy distribution and the fission-fragment mass number of ${ }^{241} \mathrm{Am}(\mathrm{n}, \mathrm{f})$ reaction.

In the processes of neutron-induced actinide nuclei fission, the excitation energy of the compound nucleus will become higher, and a few neutrons will be emitted in the fission process for de-excitation. The fission neutron spectrum of ${ }^{241} \mathrm{Am}(\mathrm{n}, \mathrm{f})$ reaction is shown in Fig. 4, which are compared with the evaluated nuclear data from ENDF/BVII.1. The comparisons show an overall good consistency, which indicate that Monte-Carlo code with the developed potential-driving model can evaluate fission neutron spectrum of ${ }^{241} \mathrm{Am}(\mathrm{n}, \mathrm{f})$ reaction on accuracy.

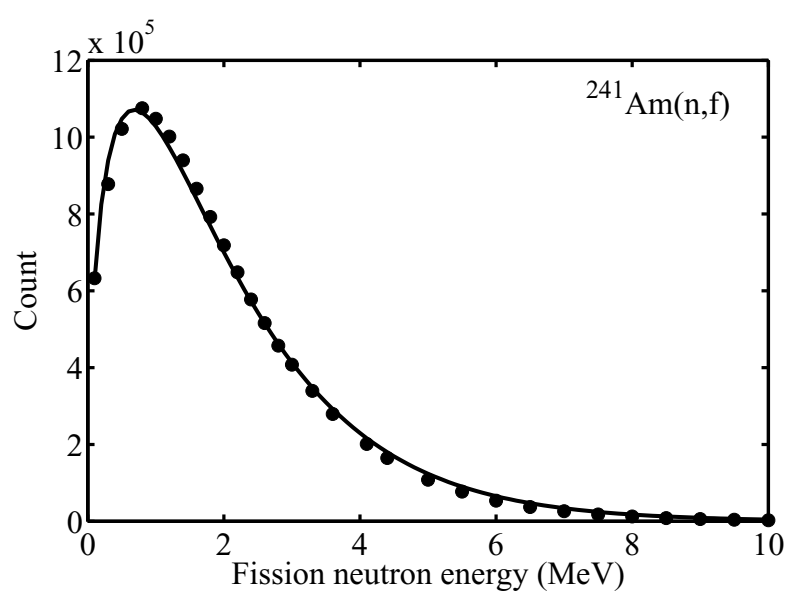

Figure 4. The fission neutrons spectrum of ${ }^{241} \mathrm{Am}(\mathrm{n}, \mathrm{f})$ reaction.
The fission-fragments nuclide is also in an excited state, which will emit characteristic $\gamma$-rays during its deexcitation process. Generally, the kind of fission products of neutron-induced actinide fission reaction can be identified by analyzing characteristic $\gamma$-rays spectrum in experiments. The typical characteristic $\gamma$-rays spectrum of ${ }^{241} \mathrm{Am}(\mathrm{n}, \mathrm{f})$ reaction is shown in Fig. 5. The structure of high-purity germanium (HPGe) coaxial detector (Model No. GMX-30190-P, produced by EG\&G ORTEC) is described accurately in the Monte Carlo simulation, with a resolution of $1.84 \mathrm{keV}$ at $1.33 \mathrm{MeV}$.

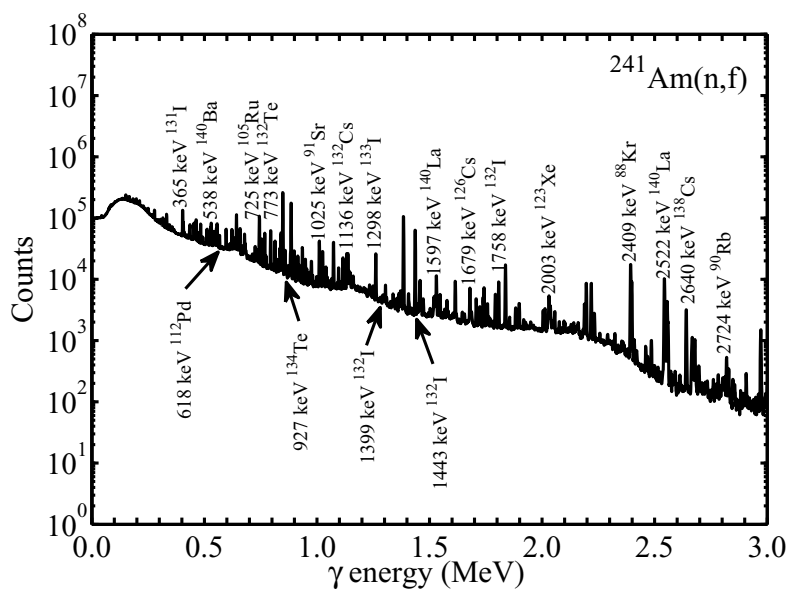

Figure 5. Typical characteristic $\gamma$-rays spectrum of ${ }^{241} \mathrm{Am}(\mathrm{n}, \mathrm{f})$ reaction.

\section{Summary and Conclusions}

The developed potential-driving model can accurately calculate the pre-neutron-emission mass distributions for neutron-induced ${ }^{241} \mathrm{Am}$ fission. The potential-driving model can well describe the variation tendency of pre-neutron-emission mass distributions with increasing incident-neutron-energy, which indicate that the potentialdriving model should provide better predictions of mass distributions for unmeasured energy regions.

Based on the developed potential-driving model, Geant4 simulates the fission process for fast neutronsinduced ${ }^{241} \mathrm{Am}$ fission, the characteristics of ${ }^{241} \mathrm{Am}(\mathrm{n}, \mathrm{f})$ reaction, such as fission-fragments yields distributions, kinetic energy distributions, fission neutron spectrum and decay $\gamma$-ray spectrum, are calculated and compared with experimental data and evaluated data. Compared with built-in G4ParaFissionModel, the calculated results from Geant 4 code with the developed potential-driving model are agree quite well with experimental data and evaluated data. The potential-driving model shows a significant advance with regard to accuracy.

Given the well predictions on the characteristics of fission reaction process, Monte-Carlo code with the developed potential-driving model can describe well the process of neutron-induced ${ }^{241} \mathrm{Am}$ fission. This work will serve for future detailed studies of neutron transmutation physics and engineering design of Accelerator-Driven Systems (ADS). 


\section{References}

[1] IAEA, Thorium fuel cycle-potential benefits and challenges, IAEA-TECDOC-1450. Austria, Vienna (2005)

[2] IAEA, Thorium based fuel options for the generation of electricity: developments in the 1990s, IAEATECDOC-1155. IAEA, Vienna (2000)

[3] E. Merle-Lucotte, D. Heuer et al., European Nuclear Conference, Brussels, Belgique (2007)

[4] S. David, A. Billebaud et al., Nucl. Instrum. Methods A 443, 510 (2000)

[5] D. Kerdraon, A. Billebaud et al., Prog. Nucl. Energy 42, 11 (2003)

[6] Y.Q. Shi, Nucl. Phys. Rev. 24, 151 (2007)

[7] C.Q. Liu, Z. Wei et al., Chinese Physics C 6, 064001 (2019)

[8] F. Vives, F.J. Hambsch, H. Bax et al., Nucl. Phys. A 662, 63 (2000)

[9] C. M. Zoller, et al., Seminar on Fission Pont dOye III, Habayla-Neuve, Belgium 56, 28(1995)

[10] F.-J. Hambsch, F. Vives, P. Siegler et al., Nucl. Phys. A 679,3 ( 2000)
[11] A. I. Sergachev, N. P. Djachenko, A. M. Kovalev et al., Sov. J.Nucl. Energy 7, 475 (1968)

[12] N. I. Akimov, V. G. Vorobeva and V. N. Kabenin, Sov. J. Nucl. Phys. 13, 31 (1971)

[13] H. Pasca, A.V. Andreev, G.G. Adamian et al., Eur. Phys. J. A 54, 32 (2018)

[14] K. Hirose, K. Nishio, S. Tanaka et al., Phys. Rev. Letters 119, 222501 (2017)

[15] K.H. Schmidt, B. Jurado, C. Amouroux, C. Schmitt, Nuclear Data Sheets 131, 107 (2016)

[16] A.J. Koning, S. Hilaire, S. Goriely, TALYS-1.8 User Manual (2015)

[17] D.M. Gorodisskiy, K.V. Kovalchuk et al., J. Korean Phys. Society 59, 919 (2011)

[18] Z. Wei, Z.E. Yao, C.L. Lan et al., J. Radioanal. Nucl. Chem. 305, 455 (2015)

[19] http://geant4.web.cern.ch/geant4/UserDocumentation/ UsersGuides/InstallationGuide/fo/BookInstalGuide.pdf, retrieved 8th December 2017

[20] A.L. Prindle, D.H. Sission et al., Physical Review C 20, 1824 (1979)

[21] R.A. Sigg, M.V. Kantrlo et al., Physical Review C 27, 245 (1983) 